\title{
Distribuição espacial de Lysiphlebus testaceipes (Cresson) (Hymenoptera: Aphidiidae) em algodoeiro cultivado em sistema adensado
}

\section{Spatial distribution of Lysiphlebus testaceipes (Cresson) (Hymenoptera: Aphidiidae) in cotton grown in a narrow system}

\author{
Tardelly de Andrade LIMA푸 José Bruno MALAQUIAS²; Jéssica Karina da Silva PACHÚ3; \\ Francisco de Sousa RAMALHO4; Renato ISIDRO5; Aline Cristina Silva LIRA ${ }^{6}$ \\ ${ }^{1}$ Mestrando do Curso de Pós-Graduação em Ciências Agrárias da Universidade Estadual da Paraíba - UEPB em parceria com \\ a Embrapa Algodão, tardellya@gmail.com; \\ ${ }^{2}$ Pós-Doutorando em Bioestatística pelo IBB-UNESP, malaquias.josebruno@gmail.com; \\ ${ }^{3}$ Doutoranda do Curso de Entomologia da ESALQ/USP, jessikapachu @gmail.com; \\ ${ }^{4}$ Pesquisador da Embrapa Algodão (Orientador): Pós-Doutorado em Entomologia, ramalhohvv@globo.com. \\ ${ }^{5}$ Professor da Universidade Federal de Campina Grande - UFCG/CDSA: Pós-Doutorado em Ciência e Tecnologia de \\ Alimentos, renatoisidro01@gmail.com. \\ ${ }^{6}$ Doutora pela Universidade Federal Rural de Pernambuco - URFPE (Programa de Pós-Graduação em Entomologia) \\ alinelira.ento@gmail.com.
}

Recebido em: 30-11-2020; Aceito em: 02-02-2021

\section{Resumo}

No presente estudo foram avaliados os efeitos do adensamento do algodoeiro na distribuição espacial do parasitoide de afídeos Lysiphlebus testaceipes (Cresson) (Hymenoptera: Aphidiidae). Os três espaçamentos adotados foram: 0,40 m x 0,20 m (E1), 0,80 m x 0,20 m (E2) e 1,60 m x 0,20 m (E3). Foi utilizado o delineamento em blocos ao acaso com quatro repetições. Os resultados evidenciaram que 0 arranjo espacial de algodão modifica o padrão de dispersão de $L$. testaceipes. O parâmetro "b" da equação de Taylor foi superior à unidade nos espaçamentos: $0,40 \mathrm{~m}$ e $1,60 \mathrm{~m}$, enquanto que em $0,80 \mathrm{~m}$ o valor de "b" foi inferior à unidade. Portanto, populações de L. testaceipes em condições de espaçamento entre linhas de $0,40 \mathrm{~m}$ e 1,60 m estão distribuídas de forma agregada, todavia se distribuindo de forma uniforme em $0,80 \mathrm{~m}$. O modelo de Iwao revelou que para os espaçamentos: $0,80 \mathrm{~m}$ e 1,60 m existe uma tendência de repulsão dos indivíduos, pois os valores da constante "a" foram menores que 1. O modelo de Nachman se ajustou apenas para os dados populacionais em algodão cultivado em $0,80 \mathrm{~m}$, portanto, para este sistema, planos que visam à estimativa do nível populacional de $L$. testaceipes podem ser baseados apenas em observações de presença/ausência, ao invés da contagem individual. Os resultados do presente estudo são relevantes para estratégias de liberação e conservação de $L$. testaceipes para controle biológico de populações de afídeos em algodão.

Palavras-chave adicionais: agregação; espaçamento; parasitoide; pulgão.

\begin{abstract}
In the present study, the effects of the densification of cotton on the spatial distribution of the aphid parasitoid Lysiphlebus testaceipes (Cresson) (Hymenoptera: Aphidiidae). The three spacings adopted were: $0.40 \mathrm{~m} \times 0.20 \mathrm{~m}$ (E1), $0.80 \mathrm{~m} \times 0.20 \mathrm{~m}$ (E2) and $1.60 \mathrm{~m} \times 0.20 \mathrm{~m}$ (E3). A randomized block design with four replications was used. The results showed that the spatial arrangement of cotton modifies the dispersion pattern of $L$. testaceipes. The parameter "b" of the Taylor equation was higher than the unit in the spacing: $0.40 \mathrm{~m}$ and $1.60 \mathrm{~m}$, while in $0.80 \mathrm{~m}$ the value of " $b$ " was lower than the unit. Therefore, populations of $L$. testaceipes in conditions of spacing between lines of $0.40 \mathrm{~m}$ and $1.60 \mathrm{~m}$ are distributed in an aggregate form, however they are uniformly distributed in $0.80 \mathrm{~m}$. The Iwao model revealed that for the spacing: $0.80 \mathrm{~m}$ and $1.60 \mathrm{~m}$ there is a trend of repulsion of individuals, since the values of the constant "a" were less than 1 . The Nachman model was adjusted only for the population data in cotton grown at $0.80 \mathrm{~m}$, therefore, for this system, plans that aim to estimate the population level of $L$. testaceipes can be based only on presence / absence observations, instead of individual counting. The present study results are relevant to strategies for the release and conservation of $L$. testaceipes for biological control of aphid populations in cotton.
\end{abstract}

Additional keywords: aggregation; aphid; parasitoid; spacing. 


\section{Introdução}

A tecnologia conhecida internacionalmente por "Ultra Narrow Row Cotton, UNRC", ou algodão em linhas ultra estreitas, também chamado de algodão "adensado", tem potencial para otimizar a produtividade e/ou precocidade e reduzir custos, por meio do aumento da produção por área cultivada (Ajayakumar et al., 2017). O adensamento da população de plantas de algodão pode melhorar a captura de luz no início do ciclo vegetativo, apesar de nem sempre resultar em aumento de produtividade devido ao impedimento da incidência de radiação nas folhas inferiores conforme fechamento do dossel (Echer \& Rosolem, 2015). A densidade de plantas de algodão também exerce influência na interação com outros fatores, tais como a incidência de plantas daninhas (Hiyat et al., 2020), e de insetos-praga juntamente com seus inimigos naturais (Malaquias et al., 2017a). Dada a importância relativa da modificação dessas interações que são mediadas pelo adensamento de plantas, é justificável a busca de informações que possam demonstrar o quanto as populações de insetos, pragas ou inimigos naturais, são afetadas (Malaquias et al., 2017a).

A amostragem permite classificar eficientemente a população para fundamentação das tomadas de decisões. Antes de se colocar em prática o plano de amostragem é necessário conhecer o padrão de dispersão dos insetos. A modelagem estatística e ecológica representa de forma simplificada a realidade de um ecossistema, sendo bastante explorados para o desenvolvimento científico e tecnológico em países de primeiro mundo (Silva et al., 2010). Interações ecológicas envolvendo indivíduos da mesma espécie e com seus inimigos naturais em um habitat específico são aspectos de capital importância para determinação das suas dinâmicas populacionais, além de elucidar com mais efetividade o padrão de dispersão. Poucos estudos têm sido aplicados ao manejo ecológico de pragas em sistema de cultivo de algodão. A distribuição espacial do inseto diz respeito à maneira pela qual este está distribuído na área, para que se possa efetivar uma amostragem mais eficiente (Silva et al., 2010). Basicamente os insetos podem distribuir-se de forma agregada, ao acaso ou ainda de forma regular. Alguns modelos de distribuição podem ser adotados tais como o método de Taylor (1961, 1984), Iwao $(1968,1975)$ e Nachman (1984).

O crescimento e o desenvolvimento do algodão (Gossypium hirsutum L.) podem ser afetados pelo ataque constante de lagartas (Malaquias et al., 2017a, 2017b, 2020) ou pulgões (Pachú et al., 2021a). Na cultura do algodão, são diversos os inimigos naturais que ocorrem predando (Pachú et al., 2018) ou parasitando (Pachú et al., 2018, 2021b) pulgões. Um destaque tem sido dado ao potencial de regulação populacional do parasitoide Lysiphlebus testaceipes (Cresson) (Hymenoptera, Braconidae, Aphidinae) (Pachú et al., 2021b). Tendo em vista que estudos sobre o padrão de dispersão de inimigos naturais de pragas constituem-se como importantes ferramentas, pelo fato de mostrar a interação entre os indivíduos de diferentes espécies e seu habitat, a pesquisa teve como objetivo avaliar os efeitos de diferentes densidades de plantas do algodoeiro na estrutura populacional do parasitoide L. testaceipes.

\section{Material e métodos}

\section{Local de instalação e delineamento experimental}

A pesquisa foi conduzida na Estação Experimental da Embrapa Algodão. A área experimental é localizada em Campina Grande - PB, na meso região do agreste paraibano, de altitude aproximada de $550 \mathrm{~m}$, 7०13'11" latitude Sul e $35^{\circ} 52^{\prime} 31^{\prime \prime}$ longitude Oeste de Greenwich.

Foi utilizado um delineamento em blocos ao acaso com quatro repetições. Os três espaçamentos adotados foram: $0,40 \mathrm{~m} \times 0,20 \mathrm{~m}$ (E1), 0,80 $\mathrm{m} \times 0,20 \mathrm{~m}$ (E2) e 1,60 m x 0,20 m (E3) (Figura 1). Após o desbaste, foram mantidas 10 plantas $/ \mathrm{m}$ de fileira. A cultivar de algodão utilizada nesse estudo foi BRS 286. As avaliações foram realizadas em 5 plantas por parcela. As unidades amostrais foram escolhidas de forma aleatória.

\section{Amostragens e Coletas de Dados:}

As determinações da distribuição e dinâmica dos insetos nas plantas de algodão foram realizadas em intervalos de sete dias, a partir da emergência das plantas. As quantificações dos insetos e suas localizações específicas foram registradas tendo como ponto de referência a localização dos nós no caule principal da planta (do nó zero ao nó terminal) e também nas folhas e estruturas frutíferas. Cada planta foi dividida em três regiões, basal ( $1 / 3$ inferior da planta), mediana (1/3 médio da planta) e apical ( $1 / 3$ superior da planta).

\section{Modelos e análise estatística}

Foram testados os seguintes modelos: Taylor (1961, 1984), Iwao (1968, 1975) e Nachman (1984). Os objetivos dos modelos de Taylor, Iwao e Nachman são de reforçar a relação existente entre a variância (б2) e a média em diferentes níveis de ocorrência dos insetos, bem como encontrar uma relação estável em função do tempo, para o desenvolvimento populacional dos inimigos naturais sob condições de campo.

\section{Resultados e discussão}

O modelo de Taylor é descrito através de uma relação existente entre a média $(\mu)$ e a variância $(\sigma 2)$ através da função exponencial: $\sigma 2=a m^{b}$ que equivale $a$ equação $\sigma 2=\ln (a)+b \ln (\mu)$. Os coeficientes: "a" e "b" são conhecidos como coeficientes de Taylor e fornecem estimativas do padrão de agregação do inseto. O coeficiente "a" é conhecido como fator de amostra- 
gem e o "b" é conhecido como índice de agregação. Quando o índice de agregação "b" é significativamente superior, igual ou inferior à unidade, o padrão espacial diz-se que é, respectivamente, agregado, aleatório e regular ou uniforme (Taylor, 1961).

De acordo com as regressões lineares envolvendo as médias e variâncias geradas para o número de pulgões parasitados por $L$. testaceipes em cada sistema de plantio, observou-se que o parâmetro "b" foi significativamente superior à unidade nos espaçamentos entre linhas: 0,40 e 1,60 m, enquanto que no espaçamento de $0,80 \mathrm{~m}$ o valor de "b" foi significati- vamente inferior à unidade (1) (Tabela 1). Portanto, o sistema de cultivo afetou o padrão de dispersão de L. testaceipes.

Bons ajustes do modelo de Taylor foram observados para todos os sistemas de cultivo. Verificou-se significância dos valores de " $F$ " em todas as condições. A variação da medida de dispersão utilizada (variância) foi descrita por $96,04,85,31$ e $89,61 \%$ dos modelos lineares em função das médias do número de insetos encontrados, nos respectivos tratamentos: 0,$40 ; 0,80 \mathrm{e}$ $1,60 \mathrm{~m}$ entre linhas. Portanto, a relação envolvendo média e variância foi linear.

Tabela 1 - Estimativa dos parâmetros do modelo de Taylor e estatísticas de análises de regressão aplicadas a populações de Lysiphlebus testaceipes em algodoeiro, em diferentes espaçamentos.

\begin{tabular}{lccrrr}
\hline Espaçamento $(\mathrm{m})$ & $\ln (\mathrm{a})$ & $\mathrm{b}$ & $\mathrm{F}$ & $\mathrm{Pr}>\mathrm{F}$ & $\mathrm{R}^{2}$ \\
\hline 0,40 & $-0,52$ & 1,76 & 148,40 & $<0,0001$ & 0,9604 \\
0,80 & $-1,23$ & 0,70 & 29,05 & 0,0030 & 0,8531 \\
1,60 & $-0,37$ & 1,82 & 70,02 & $<0,001$ & 0,8961 \\
\hline
\end{tabular}

O modelo é: $\sigma 2=a m b$ ou $\sigma 2=\ln (a)+b \ln (m)$

No presente trabalho, constatou-se que nas condições de 0,40 e 1,60 m, o padrão de dispersão deste parasitoide é agregado, portanto a agregatividade da população de $L$. testaceipes nestas condições aumentou com o aumento da média da sua ocorrência, porém sem levar os dados ao extremo (Tabela 1), enquanto no espaçamento de $0,80 \mathrm{~m}$ o valor do índice revela uma população uniforme.

Em algodoeiro cultivado no semiárido nordestino do Brasil, Bandeira et al. (2006) constaram bom ajuste do modelo de Taylor para o padrão de distribuição espacial do número de pulgões parasitadas por L. testaceipes em condições irrigadas e de sequeiro, revelando relação linear da agregação da população mediante aumento da média do número de ocorrências do parasitoide. Em sistemas de cultivo orgânico de berinjela (Solanum melongena) em Havana-Cuba, López et al., (2006) também constataram um padrão de dispersão agregado em populações de $L$. testaceipes.

Um modelo de distribuição agregado indica vastas interações intra e interespecíficas, ou envolvendo indivíduos com as condições biofísicas proporcionadas pelo agroecossistema (López et al., 2006, Lima et al., 2021). A agregatividade do parasitismo de L. testaceipsnos sistemas de cultivo adensado $(0,40 \mathrm{~m}$ e 1,60 m) pode ter sido influenciada pela agregatividade de pulgões em virtude da formação de colônias especialmente de indivíduos ápteros. De fato, Silva et al. (2010) além de observaram bons ajustes do modelo de Taylor para o hospedeiro A. gossypii, revelaram que a agregação desses pulgões em algodoeiro cultivado em sistema irrigado ou de sequeiro aumentou com a média de infestação, mas sem alcançar valores extremos.

Uma possível falta de agregação de A. gossypii em algodoeiro manejado com espaçamento entre linhas de $0,80 \mathrm{~m}$ pode ter favorecido a uniformidade de parasitismo por $L$. testaceipes, pois este endoparasitóide necessita de encontrar colônias de $A$. gossypii para se reproduzir; ou é ainda provável que a alteração na estrutura física do arranjo espacial das fileiras de algodão oferecida pelos sistemas de cultivo 0,40 e 1,60 $\mathrm{m}$ entre linhas possa ter influenciado na dinâmica de forrageio de $L$. testaceipes através de semioquímicos mediados pelas plantas (López et al., 2006). Além do mais, a arquitetura da planta pode proporcionar restrição de busca pelos parasitoides ou ofuscar partes das plantas onde os afídeos estão agregados (Stadler \& Volkl 1991).

A relação média e a variância $\left(\sigma^{2}\right)$ do modelo de Iwao é baseada no índice de aglomeração da média $\mathrm{m}^{*}$ (Lloyd, 1967). Logo: $\sigma^{2}=(a+1) m+(b-1) m^{2}$, em que "a" e " $b$ " são parâmetros da regressão entre $\mathrm{m}$ e $\mathrm{m}^{*}$ (Iwao, 1968) (Tabela 2). Conforme Iwao \& Kuno (1971), os parâmetros do modelo podem evoluir no tempo, em função da dinâmica populacional do inseto.

Tabela 2 - Estimativa dos parâmetros do modelo de Iwao e estatísticas de análises de regressão aplicadas a populações de Lysiphlebus testaceipes em algodoeiro em diferentes espaçamentos

\begin{tabular}{lllrrrr}
\hline Espaçamento & $\mathrm{n}$ & $(\mathrm{a}+1)$ & $(\mathrm{b}-1)$ & $F$ & $P r$ & \multicolumn{1}{c}{$\mathrm{R}^{2}$} \\
\hline 0,40 & 8 & 5,88 & 2,38 & 6,05 & 0,0492 & 0,5020 \\
0,80 & 7 & 0,35 & 0,02 & 1763,58 & $<0,0001$ & 0,9966 \\
1,60 & 8 & 0,76 & 5,18 & 39,41 & 0,0004 & 0,8276 \\
\hline
\end{tabular}

O modelo é: $\sigma^{2}=(a+1) m+(b-1) m^{2}$ ou $\sigma^{2} / m=(a+1) m+(b-1) m$. 
Avaliando a aplicação do modelo de Iwao para os dados populacionais de $L$. testaceipes em diferentes sistemas de cultivos, observou-se que para apenas no sistema adensado (espaçamento de 0,40 m entre linhas) se constatou baixos valores de $R^{2}$, embora 0 valor de "F" foi significativo (Tabela 4). Portanto, para o algodoeiro adensado (espaçamento 0,40 $\mathrm{m}$ entre linhas) a relação agregada média - média não se ajustou ao modelo de Iwao. O valor de "b" foi significativamente inferior à unidade no espaçamento $0,80 \mathrm{~m}$ enquanto em 1,60 m entre fileiras o índice de agregação foi significativamente superior à unidade, refletindo em padrões: uniforme e agregado, nas condições de $0,80 \mathrm{~m}$ e 1,60 m entre fileiras, respectivamente. Por outro lado, existe uma tendência de repulsão em ambas as condições, pois os valores de "a" foram < 1 (Tabela 2). Os resultados obtidos pelo modelo de Iwao para os sistemas de cultivo $0,80 \mathrm{~m}$ e $1,60 \mathrm{~m}$ confirmam os padrões de dispersão encontrados no modelo de Taylor.

A relação envolvendo média e agregado médio foi bem descrita pelo modelo linear quando nos espaçamentos $0,80 \mathrm{~m}$ e 1,60 $\mathrm{m}$ entre linhas. De fato, além dos valores de " $F$ " terem sido significativos, elevados valores de $R^{2}$ foram encontrados para esses dois arranjos de plantio. Por outro lado, Bandeira et al., (2006) e López et al., (2006) observaram que o modelo de Iwao não descreveu bem a relação média e agregado médio do número de pulgões parasitados por L. testaceips. De forma similar para populações do hospedeiro $A$. gossypii, em condições de algodoeiro em sequeiro, não foram encontrados bons ajustes deste modelo (Silva et al., 2010).

Bom ajuste do modelo de Nachman foi observado apenas para algodoeiro manejado em sistema de 0,80 m entre linhas $\left(F=12,80 ; R^{2}=0,7192 ; P=0,0159\right)$, isto implica que a relação envolvendo média de insetos e a porcentagem de plantas com ou sem ocorrência do inseto pode ser expressa adequadamente por este modelo, podendo-se desenvolver planos com objetivos que visem estimar o nível da população do parasitoide de $A$. gossypii baseado apenas em observações de presença/ausência, ao invés de contar os insetos individualmente (Tabela 3). Essa relação envolvendo as médias de ocorrência e proporção de plantas com ausência em função de médias populacionais indica que a proporção de plantas sem infestação de pulgão aumentou linearmente com o aumento médio de suas populações.

Esse comportamento dos dados deve-se possivelmente ao aumento da repulsividade da população do parasitoide no espaçamento em detrimento do crescimento populacional, deixando-se assim o espaço relativamente ocupado (Pielou, 1977). Para a joaninha Scymnus sp., Pachú et al. (2014) registraram o não ajuste dos dados ao modelo de Nachman, revelando que planos de amostragem para Scymnus sp. não podem ser baseados na ausência/presença dos insetos, implicando na necessidade de contagem dos predadores individualmente.

Tabela 3 - Estimativa dos parâmetros do modelo de Nachman e estatísticas de análises de regressão aplicadas a populações de Lysiphlebus testaceipes em algodoeiro em diferentes espaçamentos

\begin{tabular}{lllllll}
\hline Espaçamento & $\mathrm{n}$ & $\ln (\mathrm{a})$ & $\mathrm{b}$ & $\mathrm{F}$ & $\mathrm{Pr}>\mathrm{F}$ & $\mathrm{R}^{2}$ \\
\hline 0,40 & 8 & $\ln (-1,0423)$ & 0,3412 & $5,68 \mathrm{~ns}$ & 0,0545 & 0,4863 \\
0,80 & 7 & $\ln (-1,3939)$ & 0,1587 & 12,80 & 0,0159 & 0,7192 \\
1,60 & 8 & $\ln (-0,8196)$ & 0,056 & $0,14 \mathrm{~ns}$ & 0,7163 & 0,0201 \\
\hline
\end{tabular}

O modelo é: $\ln (-\ln (\mathrm{p} 0)=\ln (a)+b \ln (m)$

A adoção de métodos quantitativos que permitem uma efetiva inferência sobre a dinâmica populacional de pragas e de seus inimigos naturais é fundamental para o estabelecimento de programas de conservação e liberação de inimigos naturais (Pachú et al., 2021b, Lima et al., 2021). L. testaceipes é de extrema relevância para controle populacional de $A$. gossypii em algodão, especialmente quando seu hospedeiro se encontra em altas densidades (Malaquias et al., 2017a).

\section{Conclusão}

Os resultados deste estudo são importantes para estratégias de liberação e conservação de $L$. testaceipes, visando, o controle de $A$. gossypii em algodoeiro cultivado em diferentes espaçamentos, e pode-se concluir que:

(1) $O$ arranjo espacial de fileiras de algodão modifica o padrão de dispersão do parasitoide L. testaceipes.

(2) Populações de L. testaceipes em condições de algodão adensado, em espaçamento de 0,40 m e 1,60 m estão distribuídas de forma agregada, enquanto no espaçamento de $0,80 \mathrm{~m}$ distribuem-se de forma uniforme.

(3) Planos visando à estimativa do nível populacional do parasitoide $L$. testaceipes podem ser baseados apenas em observações de presença/ausência, ao invés de contar os insetos individualmente somente em algodão cultivado em 0,80 m entre fileiras.

\section{Referências}

Ajayakumar MY, Umesh MR, Shivaleela S, Nidagundi JM (2017) Light interception and yield response of cotton varieties to high density planting and fertilizers in sub-tropical India. Journal of Applied and Natural Science 9(3): 1835-1839.

Bandeira CM, Wanderley PA, Ramalho FS, Silva GF, Silva JPS, Malaquias JB, Pereira AIA, Zanuncio JC (2006) Spatial models of distribution of Lisiphlebus testaceips (Cresson) (Hymenoptera: Braconidae) a parasitic wasp of Aphis gossipii Glover on cotton. In: XXI Congresso de Entomologia, 2006. Recife v.1. 
Echer FR, Rosolem CA (2015) Cotton yield and fiber quality affected by row spacing and shading at different growth stages. European Journal of Agronomy 65: 18-26.

Hiyat S, Hussain N, Rehmani MIA, Abbas MN, Raza S, Dar JS, Yasir TA (2020) Effect of growth regulator and ultra-narrow row (UNR) spacing on growth, yield and yield components of cotton. Pakistan Journal of Agricultural Research 33(3): 561.

Iwao $S$ (1968) A new regression method for analyzing the aggregation pattern of animal populations. Research Population Ecology 10: 1-20.

Iwao S (1975) A new method of sequential sampling to classify populations relative to a critical density. Research Population Ecology 16:281-288.

Iwao S, Kuno E (1971) Use of the regression of mean crowding on mean density for estimating sample size and the transformation of data for the analysis of variances. Research Population Ecology 10: 210-214.

Lima TA, Malaquias JB, Pachú, JKS, Ramalho FS (2021) Patrón de la distribución espacial de los enemigos naturales: subsidios para programas de muestreo. In: Pachu, JKS, Malaquias JB, Ramalho FSR (eds) Métricas poblacionales aplicadas a estudios de poblaciones de enemigos naturales: Casos en algodón agroecológico. Editorial Académica Española, p. 30-43.

López M, Miranda I, Fernandez M (2006) Spatial patterns and ability of Lysiphlebus testaceipes as a natural control of Aphis gossypii in eggplant. Revista de Protección Vegetal 21: 22-26.

Lloyd M 1967 Mean crowding. Journal of Animal Ecology 36: 1-30.

Malaquias JB, Ramalho FS, Dias CTDS, Brugger BP, Lira ACS, Wilcken CF, Pachú JKS, Zanuncio JC (2017a) Multivariate approach to quantitative analysis of Aphis gossypii Glover (Hemiptera: Aphididae) and their natural enemy populations at different cotton spacings. Scientific Reports 7(1): 1-11.

Malaquias JB, Godoy WA, Garcia AG, Ramalho FDS, Omoto C (2017b) Larval dispersal of Spodoptera frugiperda strains on Bt cotton: a model for understanding resistance evolution and consequences for its management. Scientific Reports 7(1): 1-10.

Malaquias JB, Caprio MA, Godoy WA, Omoto C, Ramalho FS, Pachú JK (2020) Experimental and theoretical landscape influences on Spodoptera frugiperda movement and resistance evolution in contaminated refuge areas of Bt cotton. Journal of Pest Science 93(1): 329-340.

Malaquias JB, Godoy WAC, Caprio MA, Pachú JKS, Ramalho FS, Omoto C, Ferreira CP (2021) Evolutionary process modelling with Bayesian inference of Spodoptera frugiperda ballooning and walking dispersal on $\mathrm{Bt}$ and non-Bt cotton plants mixture. Entomologia Experimentalis et Applicata.

https://doi.org/10.1111/eea.13045
Mesina RRV (1986) Disposição espacial de Panonychusulmi (Koch, 1836) (Acarina: Tetranychidae) e determinação do número de amostras na macieira. $88 \mathrm{f}$. Dissertação (Mestrado em Entomologia) Universidade Federal do Paraná, Curitiba.

Nachman G (1984) Estimates of mean population density and spatial distribution of Tetranchusurticae (Acarina:Tetranychidae) and Phytoseiulus persimilis (Acarina: Phytoseiidae) based upon the proportion of empty sampling units. Journal of Applied Ecology 21: 903-913.

Pachú JK, Ramalho FS, Andrade TL, Malaquias JB, Santos BDB, Lira ACS (2014) Padrão de distribuição espacial de Scymnus sp. em algodoeiro cultivado em sistema "ultra-narrow-row cotton". Anais do XXV Congresso Brasileiro de Entomologia, Goiânia.

Pachú JKS, Malaquias JB, Godoy WA, Ramalho FS, Almeida BR, Rossi F (2018). Models to describe the thermal development rates of Cycloneda sanguinea $\mathrm{L}$. (Coleoptera: Coccinelidae). Journal of Thermal Biology 73: 1-7.

Pachú JKS, Macedo FC, da Silva FB, Malaquias JB, Ramalho FS, Oliveira RF, Godoy W A (2021a) Imidacloprid-mediated stress on non-Bt and Bt cotton, aphid and ladybug interaction: approaches based on insect behaviour, fluorescence, dark respiration and plant electrophysiology. Chemosphere, 263: 127561.

Pachú, JKS, Lima TA, Malaquias JB, Ramalho FS (2021b) Diversidad de enemigos naturales en algodón agroecológico. In: Pachu, JKS, Malaquias JB, Ramalho FSR (eds) Métricas poblacionales aplicadas a estudios de poblaciones de enemigos naturales: Casos en algodón agroecológico. Editorial Académica Española, p.03-29.

Pielou EC (1977) Mathematical ecology. John Wiley. 302p.

Stadler B, VölkI W (1991) Foraging patterns of two aphid parasitoids Lysiphlebus testaceipes and Aphidius colemani on banana. Entomologia Experimentalis et Applicata 58:221-230.

Silva GF, Ramalho FS, Pereira AIA, Nunes Júnior ES, Pereira RG (2010) Padrão de distribuição temporal de Aphis gossypii em algodoeiro irrigado e de sequeiro no estado do Ceará. Revista Verde de Agroecologia e Desenvolvimento Sustentável 5:195-203.

Taylor LR (1961) Aggregation, variance and the mean. Nature 189:732-735.

Taylor LR (1984) Assessing and interpreting the spatial distributions of insect populations. Annual Review of Entomology 29:321-357. 